PHYSICAL REVIEW D 93, 039904(E) (2016)

\title{
Erratum: Nucleon and pion structure with lattice QCD simulations at physical value of the pion mass [Phys. Rev. D 92, 114513 (2015)]
}

\begin{abstract}
A. Abdel-Rehim, C. Alexandrou, M. Constantinou, P. Dimopoulos, R. Frezzotti, K. Hadjiyiannakou, K. Jansen, Ch. Kallidonis, B. Kostrzewa, G. Koutsou, M. Mangin-Brinet, M. Oehm, G. C. Rossi, C. Urbach, and U. Wenger (Received 29 January 2016; published 10 February 2016)
\end{abstract}

We present results on the nucleon scalar, axial, and tensor charges as well as on the momentum fraction and the helicity and transversity moments. The pion momentum fraction is also presented. The computation of these key observables is carried out using lattice QCD simulations at a physical value of the pion mass. The evaluation is based on gauge configurations generated with two degenerate sea quarks of twisted mass fermions with a clover term. We investigate excited state contributions with the nucleon quantum numbers by analyzing three sink-source time separations. We find that, for the scalar charge, excited states contribute significantly and, to a lesser degree, for the nucleon momentum fraction and the helicity moment. Our result for the nucleon axial charge agrees with the experimental value. Furthermore, we predict a value of $1.027(62)$ in the $\overline{\mathrm{MS}}$ scheme at $2 \mathrm{GeV}$ for the isovector nucleon tensor charge directly at the physical point. The pion momentum fraction is found to be $\langle x\rangle_{u-d}^{\pi^{ \pm}}=0.214(15)\left(\begin{array}{c}+12 \\ -9\end{array}\right)$ in the $\overline{\mathrm{MS}}$ at $2 \mathrm{GeV}$.

DOI: 10.1103/PhysRevD.93.039904
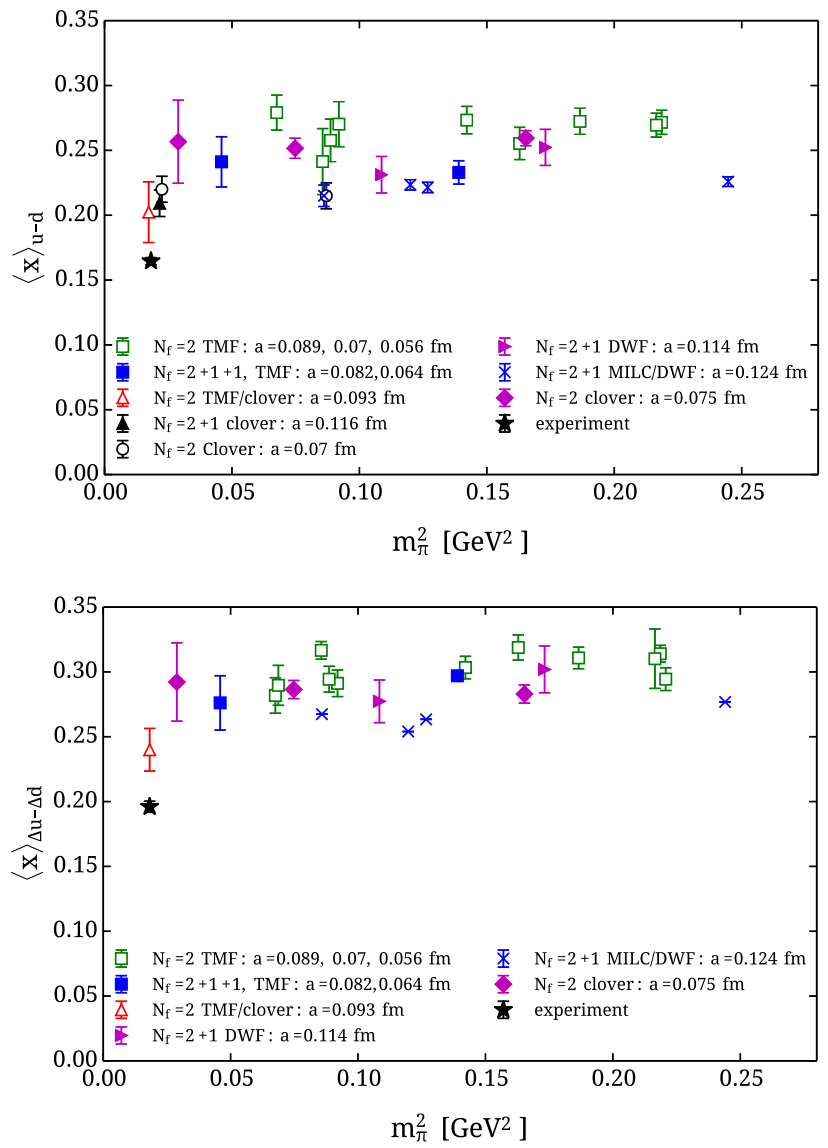

FIG. 1. Isovector nucleon momentum fraction $\langle x\rangle_{u-d}$ and helicity $\langle x\rangle_{\Delta u-\Delta d}$. Twisted mass fermion results are shown for $N_{f}=2$ ensembles (open green squares), for two $N_{f}=2+1+1$ ensembles (blue iller square) and for the physical ensemble with a clover term (open red triangle) taken from Table VI. Also shown are results from RBC-UKQCD using $N_{f}=2+1$ DWF (magenta right pointing triangle) [1], from LHPC using DWF on $N_{f}=2+1$ staggered sea (blue crosses) [2] and QCDSF/UKQCD using $N_{f}=2$ clover fermions (filled magenta diamond) [3]. For $\langle x\rangle_{u-d}$ we also show results from LHPC using $N_{f}=2+1$ clover with 2-HEX smearing (filled black triangles) [4] and $N_{f}=2$ clover (open black circle) [5]. All values are extracted using the plateau method and $t_{s} \sim(1-1.2)$ fm, except our result at the physical point for which $t_{s} \sim 1.3 \mathrm{fm}$ was used. The experimental value for $\langle x\rangle_{u-d}$ is taken from Ref. [6] and for $\langle x\rangle_{\Delta u-\Delta d}$ from Ref. [7]. 
With this erratum, we show the corrected experimental value of the helicity in Fig. 1. This figure replaces Fig. 17 of the published paper.

[1] Y. Aoki, T. Blum, H.-W. Lin, S. Ohta, S. Sasaki, R. Tweedie, J. Zanotti, and T. Yamazaki, Phys. Rev. D 82, 014501 (2010).

[2] J. D. Bratt et al. (LHPC Collaboration), Phys. Rev. D 82, 094502 (2010).

[3] D. Pleiter et al. (QCDSF/UKQCD Collaboration), Proc. Sci., LATTICE (2010) 153.

[4] J.R. Green, M. Engelhardt, S. Krieg, J. W. Negele, A. V. Pochinsky, and S. N. Syritsyn, Phys. Lett. B 734, 290 (2014).

[5] G. S. Bali, S. Collins, B. Gläßle, M. Göckeler, J. Najjar, R. H. Rödl, A. Schäfer, R. W. Schiel, A. Sternbeck, and W. Söldner, Phys. Rev. D 90, 074510 (2014).

[6] S. Alekhin, J. Blumlein, and S. Moch, Phys. Rev. D 86, 054009 (2012).

[7] J. Blumlein and H. Bottcher, Nucl. Phys. B841, 205 (2010). 